\title{
Addition of dianions of carboxylic acids to imines. Influence of the acid in the outcome of the reaction
}

\author{
Salvador Gil, ${ }^{\text {a,b }}$ Margarita Parra, ${ }^{\text {a,b, }}{ }^{*}$ Pablo Rodriguez ${ }^{\mathrm{a}}$ \\ ${ }^{a}$ Department of Organic Chemistry. Universidad de Valencia, 46100-Burjassot. Spain \\ ${ }^{b}$ Instituto de Reconocimiento Molecular y Desarrollo Tecnológico. Centro Mixto Universidad \\ Politécnica de Valencia-Universidad de Valencia. Spain \\ E-mail: margarita.parra@uv.es
}

\begin{abstract}
The addition of different carboxylic acids dianions to $N$-benzylidenebenzeneamine have been studied. The outcome of the reaction depends on the acid. Saturated ones lead to $\beta$-aminoacids with good yields and syn-selectivity whereas $\alpha, \beta$-unsaturated ones lead to $\alpha-$ and $\gamma-$ regioisomers with a regioselectivity that depends on the steric hindrance around the reactive centre. From some of these unsaturated acids, polyunsaturated carboxylic acids can be obtained as a change in the reaction conditions lead to the in situ elimination of aniline. From o-methyl aromatic acids $\delta$-aminoacids are isolated with uneven results. Those derived from six member arene carboxylic acids give, on standing, dihydro-2-pyridones as the sole product.
\end{abstract}

Keywords: Dianions, imines, regioselectivity, diastereoselectivity

\section{Introduction}

Carboxylic acids are synthetically useful building blocks because, after double deprotonation, they afford enediolates that react with several electrophiles under appropriate conditions. ${ }^{1}$ Lithium dialkylamides are generally used as bases to generate lithium enediolates due to their strength and low nucleophilicity combined with their solubility in non-polar solvents. ${ }^{1-2}$ It is well established that lithium enolates exist as complex ion pair aggregates in these solvents whose metal centre may be coordinated to solvent molecules or to other chelating ligands, such as the amines resulting from a deprotonation of the acid by the lithium amide. The data available so far confirm the complexity present in these aggregated reactive species, whose reactivity and selectivity can be influenced by several factors. ${ }^{3}$ From our experience in the reactivity of dianions of carboxylic acids with several eletrophiles can be concluded that an optimization study for each new reaction is required. 
In recent years, there has been an increasing interest in the synthesis of $\beta$-lactams ${ }^{4}$ or $\beta$ aminoacids and esters ${ }^{5}$ as they are useful building blocks for the synthesis of $\beta$-peptides that are present in several potent drugs. ${ }^{6}$ Several methods are available for the synthesis of $\beta$-amino acids but one of the most versatile are the additions of carbon nucleophiles to imine equivalents (imines, nitrones or oximes) in Reformatsky or Mannich-type reactions . ${ }^{7}$ In this context, we think that we can study the reactivity of the dianions of carboxylic acids using imines as electrophiles, despite that we have previously described a synthesis of $\beta$-aminoacids from the same methodology but using isocyanates as electrophiles. ${ }^{8}$

Some authors have studied the addition to imines from different enolates: esters ${ }^{7,9,10}$ or amides ${ }^{11}$ that render opposite diastereoisomer outcome (Scheme 1). Only one reference has been found on the reactivity of phenylacetic dianion with N-benzylidenebenzenamine with a short study of the influence of the cation. ${ }^{12}$

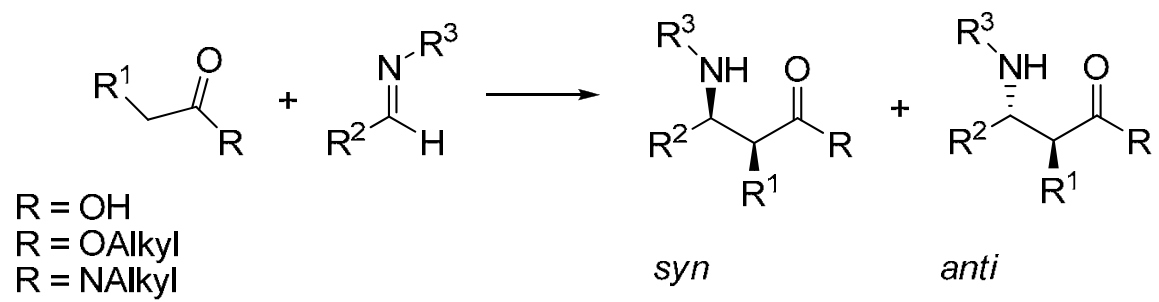

Scheme 1. General reactivity of enolates with imines.

In general, moderate yields are described with a diastereoselectivity strongly dependent on the enolate. Thus, lithium enolates from menthyl esters ${ }^{9}$ and titanium enolates from alkyl ${ }^{7}$ or $\alpha$ phenylseleno-alkyl ${ }^{10}$ esters afford the syn-diastereoisomer as major product. In contrast ethylzinc enolates derived from 4-acryloylmorpholine lead to the anti-product in around $70 \%$ d.e. ${ }^{11}$

\section{Results and Discussion}

Based upon these results, we have examinated the scope of the addition of carboxylic acids dianions to $N$-benzylidenebenzenamine (1). In a first step, saturated carboxylic acids were tested and the results are summarized in Table 1.

The dianion is generated by treating the corresponding acid with lithium dialkylamine. Previous studies by our group on the reactivity of enediolates led us to develop different conditions for the generation of the dianions of carboxylic acids which, in some cases, improve the yield and selectivity of the reaction. ${ }^{13}$ Thus, both the nature and amount of the amine can be changed, a process that is usually necessary in order to optimize the reaction conditions as it is not always possible to predict the best conditions for each electrophile. As can be seen in the table, both lithium diethylamide (less hindered and more basic) and lithium cyclohexyl-isopropylamide (Cyi-PrNLi) yields good results in the addition to imines (see entries 2 and 3 table 1). Lithium 
diethylamide has been used throught the study as it is more accesible and leads to a simpler workup.

Table 1. Addition of lithium enediolates of carboxylic acid to $N$-benzylidenebenzenamine

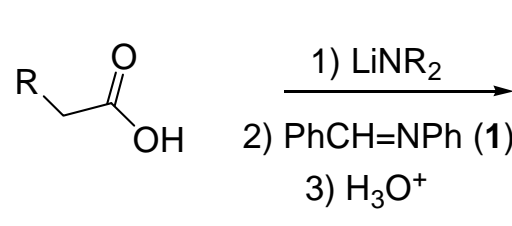<smiles>[R]C(C(=O)O)[C@H](Nc1ccccc1)[C@@H](Nc1ccccc1)C([R])C(=O)O</smiles>
(2) $\mathrm{R}=\mathrm{Ph}$
(3) $\mathrm{R}=\mathrm{CH}_{2} \mathrm{Ph}$
(5) $\mathrm{R}=\mathrm{Ph}$
(7) $\mathrm{R}=\mathrm{CH}_{2} \mathrm{Ph}$
(6) $\mathrm{R}=\mathrm{Ph}$
(4) $\mathrm{R}=\mathrm{CH}_{2} \mathrm{CH}_{2} \mathrm{CH}_{3}$
(9) $\mathrm{R}=\mathrm{CH}_{2} \mathrm{CH}_{2} \mathrm{CH}_{3}$
(8) $\mathrm{R}=\mathrm{CH}_{2} \mathrm{Ph}$
(10) $\mathrm{R}=\mathrm{CH}_{2} \mathrm{CH}_{2} \mathrm{CH}_{3}$

\begin{tabular}{cccccccc}
\hline Entry & Acid & $\begin{array}{c}\text { Eq. } \\
\text { Imine }\end{array}$ & Base* & $\mathrm{T}$ & Yield (\%) & \multicolumn{2}{c}{ diastereoselectivity } \\
& & & & & Syn & Anti \\
\hline 1 & $\mathbf{4}$ & 1 & $\mathrm{Cyi}-\mathrm{PrNLi}$ & $-50^{\circ} \mathrm{C}$ & 49 & 72 & 28 \\
2 & $\mathbf{4}$ & 1 & $\mathrm{Cyi}-\mathrm{PrNLi}$ & r.t. & 57 & 70 & 30 \\
3 & $\mathbf{4}$ & 1 & $\mathrm{Et}_{2} \mathrm{NLi}$ & r.t. & 56 & 73 & 27 \\
4 & $\mathbf{4}$ & 3 & $\mathrm{Et}_{2} \mathrm{NLi}$ & r.t. & 74 & 80 & 20 \\
5 & $\mathbf{4}$ & 3 & $\mathrm{Et}_{2} \mathrm{NLi}$ & r.t. & 18 & 72 & 28 \\
6 & $\mathbf{3}$ & 3 & $\mathrm{Et}_{2} \mathrm{NLi}$ & r.t. & 70 & 71 & 29 \\
7 & $\mathbf{2}$ & 3 & $\mathrm{Et}_{2} \mathrm{NLi}$ & r.t. & 65 & 100 & 0 \\
\hline
\end{tabular}

* 2.2 equivalents except for entry 5 where a sub-stoichiometric amount ( 0.5 equiv.) was used.

It is well known that some dianions of carboxylic acids undergo an easy reprotonation by the amine while others do not but it is no easy to predict the behaviour of a particular acid due to the aggregation nature of these complex systems as mentioned above. We think that this is a crucial factor to determine whether sub-stoichiometric (0.5 equiv.) or equimolecular (4.5 equiv.) amounts of amine (entries 4 and 5), always with an equimolecular amount of BuLi, lead to higher yields.

From the optimization it can be concluded that the general conditions in order to complete these reactions are addition of 3 eq of imine (1) and 21 hours of reaction time at room temperature.

The syn-anti assignation for each diastereoisomer was made by nOe experiments and the ratios were determinded by means of integration of the ${ }^{1} \mathrm{H}$ NMR signal corresponding to the $\mathrm{CH}-$ $\mathrm{N}$. The syn diastereoselectivity has been previously explained. It can be rationalized by considering an $E$ geometry for the imine and that the TS-1, shown in Figure 1, would be more favorable. . $^{7,10}$ 


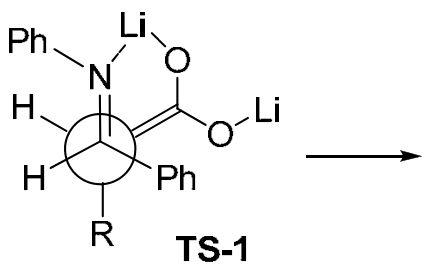

TS-1

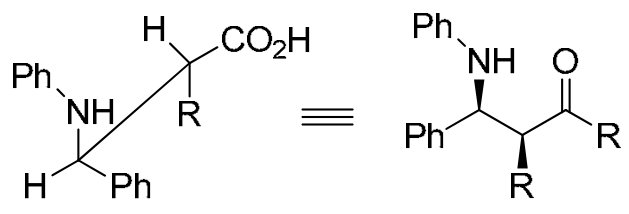

syn diastereoisomer

Figure 1. Favorable approach model.

This approach agrees with our results for phenyl acetic acid $(\mathrm{R}=\mathrm{Ph}, 2)$ where a $\pi$-stacking interaction between the enolate system and the phenyl group at the imine carbon atom would lead to TS-1 being a highly favorable approach and, therefore, the syn diastereoisomer as the sole product (table 1 , entry 7 ).

$\beta$-Aminoacids can be considered intermediates to the biologically important $\beta$-lactams as several methods for its cyclization have been described. ${ }^{10,14}$ We have extended the procedure to $\alpha, \beta$-unsaturated carboxylic acids (Table 2). It is well known that those dienediolates behave as ambident nucleophiles, through either their $\alpha$ or $\gamma$ carbon atoms, leading to single or predominant adducts when allowed to react with electrophiles under adequate conditions. ${ }^{1,15}$ Thus, $\alpha$-attack is favored for irreversible reactions whereas $\gamma$-adducts are obtained on addition to carbonyl compounds and nitriles under equilibrium conditions. ${ }^{15,16}$ When the dienediolate from tiglic acid 12 reacts with 1 under the optimized conditions described above for saturated carboxylic acids, only $\gamma$-adduct is obtained with low yield as around $29 \%$ starting acid is recovered (Table 2, entry 1). Thus, a new optimization of the reaction conditions was required for this acid. Results are summarized in table 2 . In a similar study as that described above, we have modified the amine and the temperature in order to optimize the regioselective obtention of the $\gamma$-adduct and we found that with cyclohexylisopropylamine as base and 21 hours of reaction time at $-50^{\circ} \mathrm{C}$, only 1 eq. of imine is required to attain the best results. It is worth to note that elimination of aniline from the $\gamma$-adduct 18 is promoted either by rising the temperature or by using sub-stoichiometric amounts of amine, leading to $\mathbf{2 1}$ as a major or even exclusive product.

Crotonic acid (11) yields a mixture of products. From its spectral data, the formation of $\gamma-$ and $\alpha$-adducts along with polyunsaturated polymerization products could be deduced. On methylation, only $\gamma$-adduct was obtained in the neutral fraction as methyl ester.

3,3-Dimethylacrylic acid (13) yields only one diastereoisomer of the $\alpha$-adduct that was assigned by nOe experiments as above. A small amount of $\gamma$-adduct was also observed in the crude reaction mixture but it could not be isolated. The formation of only one diastereoisomer of the $\alpha$ adduct can be explained as above (for phenyl acetic acid) by considering a $\pi$-stacking between phenyl and vinyl moieties rendering the approach depicted in Figure 1 more favorable. 
Table 2. Addition of $N$-benzylidenebenzeneamine to dienediolates from unsaturated carboxylic acids

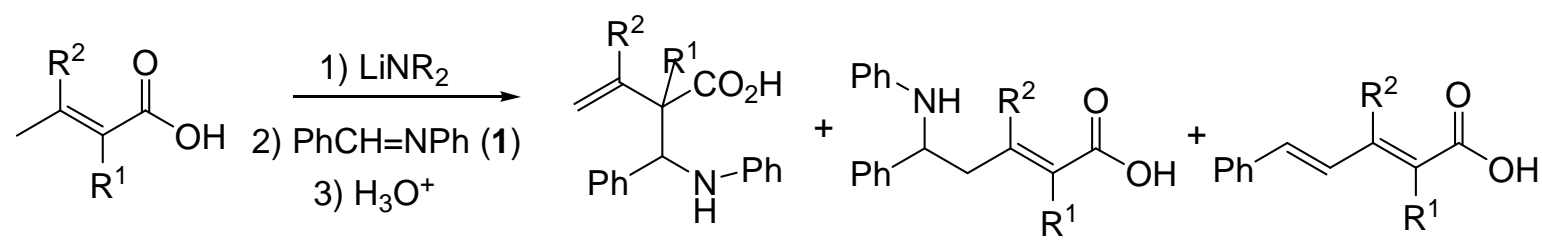
(11) $\mathrm{R}^{1}=\mathrm{R}^{2}=\mathrm{H}$
(14) $\mathrm{R}^{1}=\mathrm{R}^{2}=\mathrm{H}$
(17) $\mathrm{R}^{1}=\mathrm{R}^{2}=\mathrm{H}$
(20) $R^{1}=R^{2}=H$
(12) $\mathrm{R}^{1}=\mathrm{CH}_{3} ; \mathrm{R}^{2}=\mathrm{H}$
(15) $\mathrm{R}^{1}=\mathrm{CH}_{3} ; \mathrm{R}^{2}=\mathrm{H}$
(18) $\mathrm{R}^{1}=\mathrm{CH}_{3} ; \mathrm{R}^{2}=\mathrm{H}(\mathbf{2 1}) \mathrm{R}^{1}=\mathrm{CH}_{3} ; \mathrm{R}^{2}=\mathrm{H}$
(13) $\mathrm{R}^{1}=\mathrm{H} ; \mathrm{R}^{2}=\mathrm{CH}_{3}$
(16) $\mathrm{R}^{1}=\mathrm{H} ; \mathrm{R}^{2}=\mathrm{CH}_{3}$
(19) $\mathrm{R}^{1}=\mathrm{H} ; \mathrm{R}^{2}=\mathrm{CH}_{3}$ (22) $\mathrm{R}^{1}=\mathrm{H} ; \mathrm{R}^{2}=\mathrm{CH}_{3}$

\begin{tabular}{|c|c|c|c|c|c|c|c|c|}
\hline \multirow[t]{2}{*}{ Entry $^{a}$} & \multirow[t]{2}{*}{ Acid } & \multirow[t]{2}{*}{ Base $^{b}$} & \multirow[t]{2}{*}{$\mathrm{T}\left({ }^{\circ} \mathrm{C}\right)$} & \multirow[t]{2}{*}{ Yield (\%) } & \multirow{2}{*}{$\begin{array}{l}\text { Starting } \\
\text { acid }(\%)\end{array}$} & \multicolumn{3}{|c|}{ Products } \\
\hline & & & & & & $\alpha$ & $\gamma$ & $-\mathrm{NH}_{2} \mathrm{Ph}$ \\
\hline 1 & 12 & $\mathrm{Et}_{2} \mathrm{NLi}$ & -78 & 46 & 29 & & 71 & \\
\hline 2 & 12 & $\mathrm{Et}_{2} \mathrm{NLi}$ & -50 & 53 & & & 100 & \\
\hline 3 & 12 & $\mathrm{Et}_{2} \mathrm{NLi}^{\mathrm{c}}$ & -50 & 40 & & & 23 & 77 \\
\hline 4 & 12 & AZA-Li ${ }^{\mathrm{d}}$ & -50 & 50 & 27 & & 73 & \\
\hline 5 & 12 & $\mathrm{Et}_{2} \mathrm{NLi}$ & r.t. & 70 & & & & 100 \\
\hline 6 & 12 & Cyi-PrNLi & -50 & 76 & & & 100 & \\
\hline 7 & 11 & Cyi-PrNLi & -50 & $43^{\mathrm{e}}$ & & & & \\
\hline 8 & 13 & Cyi-PrNLi & -50 & 54 & & 100 & & \\
\hline
\end{tabular}

a 1 equivalent of imine has been used in all entries except for entry 1 where 3 equivalents of imine have been used.

b 4.5 equivalents.

${ }^{\mathrm{c}}$ Sub-stoichiometric amount 0.5 equivalents.

${ }^{\mathrm{d}} \mathrm{N}$-lithium-1,3,3-trimethyl-6-azabicyclo[3.2.1]octane.

${ }^{\mathrm{e}}$ As a complex mixture. From it, pure $\gamma$-adduct can be isolated as methyl ester.

Results concerning the formation of $\alpha$ - or $\gamma$-adducts can be rationalized by the relative stability of the products or via a competing chair Zimmerman-Traxler model depicted in Figure 2 , similar to that described by Von Koten et al. ${ }^{17}$ for ester enolates. Usually the $\gamma$-adducts are more stable from a thermodynamically point of view, thus $\mathbf{1 1}$ and $\mathbf{1 2}$ led to products. For the acid 13 a strong steric hindrance in TS-2 between $\mathrm{Ph}$ and $\mathrm{Me}$ groups could render this approach unfavorable and despite the fact that a small amount of $\gamma$-adduct can be observed, the major product is the $\alpha$-adduct probably through a TS-3 approach. The elimination process is difficult to rationalize under the strong basic media of the reaction. The same authors describe a polymerization process when the reaction temperature arise over $-20^{\circ} \mathrm{C} .^{17}$ 


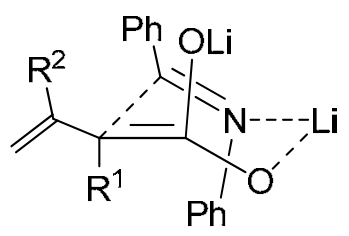

TS-2

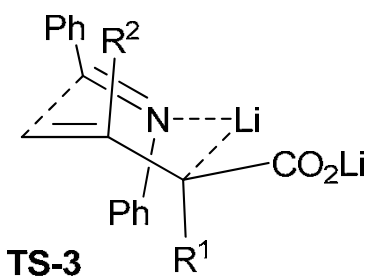

TS-3

Figure 2. Zimmerman-Traxler model for the obtention of $\alpha$ - or $\gamma$-adducts in reaction of dienediolates.

We have extended this methodology to dianions derived from ortho-methylarenic acids (Scheme 2). A new optimization was required to get the best reaction conditions. These are similar to those described above for $\alpha, \beta$-unsaturated acids but yields were highly dependent on the acid. The most noticeable result is the spontaneous cyclization to the corresponding dihydro2-pyridone of those $\delta$-aminoacids derived from six-member ring aromatic acids. Thus, whereas aminoacids $\mathbf{3 1}$ and $\mathbf{3 2}$ are stable enough to keep them unchanged for a long time, 29 and $\mathbf{3 0}$ lead to the dihydropyridone within two days and cyclization of $\mathbf{2 8}$ is so quick that only $\mathbf{3 3}$ could be characterized.<smiles>Cc1[X]cccc1C(=O)O</smiles>

(23) $\mathrm{X}=\mathrm{CH}$

(24) $X=N$

(25)<smiles>[X]c1cccc(C)c1C(=O)O</smiles>

(26)<smiles>Cc1ccsc1C(=O)O</smiles>

(27)<smiles>Cc1cc(C(=O)O)c(C)o1</smiles>

1) Base

2) $\mathrm{PhCH}=\mathrm{NPh}$

3) $\mathrm{H}_{3} \mathrm{O}^{+}$
(28) $\mathrm{X}=\mathrm{CH}$

(29) $X=N$<smiles>[X]c1cccc(C(=O)O)c1CC(Nc1ccccc1)c1ccccc1</smiles>

(30)<smiles>O=C(O)c1ncccc1CC(Nc1ccccc1)c1ccccc1</smiles>

(35)

(33) $\mathrm{X}=\mathrm{CH} \quad 48 \%$

(34) $X=N \quad 76 \%$<smiles>[X]c1cccc2c(=O)n(-c3ccccc3)c(-c3ccccc3)cc12</smiles><smiles>O=C1c2ncccc2CC(c2ccccc2)N1c1ccccc1</smiles>

(31)<smiles>O=C(O)c1sccc1CC(Nc1ccccc1)c1ccccc1</smiles>

(32)

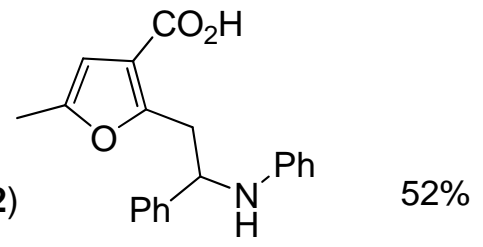

Scheme 2. Addition of $N$-benzylidenebenzeneamine to dianions from $O$-methylarenic acids. 


\section{Conclusions}

In conclusion, we have studied the reactivity of dianions from carboxylic acids with $\mathrm{N}$ benzylidenebenzeneamine. We have found that the outcome of the reaction have a critical dependence on the acid. Saturated ones lead to $\beta$-aminoacids with good yields and synselectivity. With unsaturated carboxylic acids two regioisomers are possible and the regioselectivity depens on the steric hindrance around the reactive centre. From o-methyl aromatic acids $\delta$-aminoacids are isolated with uneven results. Those derived from six-membered arene carboxylic acids give, on standing, dihydro-2-pyridones as the sole product.

\section{Experimental Section}

General Procedures. Melting points were determined with a Cambridge Instruments Hot Plate Microscope and are uncorrected. IR spectral data were obtained for liquid film or $\mathrm{KBr}$ discs; the measurements were carried out by the SCSIE (Servei Central de Suport a la Investigació Experimental de la Universitat de Valencia) on a Matteson Satellite FTIR 3000 model Spectrophotometer. NMR spectra were recorded for solutions, in the stated solvent, with a Bruker Avance 300, 400 or 500 spectrometers. High resolution mass spectra were determined with a Fison VG Autospec spectrometer. Flash Column Silica Gel of 230-400 mesh (manufacturer: Scharlau) was used for flash column chromatography, with hexane/ethyl acetate mixtures for elution. All reactions were carried out under argon atmosphere, using standard conditions for exclusion of moisture, in oven dried glassware, in THF freshly distilled from blue benzophenone ketyl and with amines distilled from $\mathrm{CaH}_{2}$ and stored over molecular sieves and kept under Ar. The BuLi used was $1.6 \mathrm{M}$ in hexane. Exact determination of the solution's concentration was periodically checked before use. The reaction temperature $\left(-78^{\circ} \mathrm{C}\right)$ was achieved by cooling with a $\mathrm{CO}_{2} /$ acetone bath and $0^{\circ} \mathrm{C}$ achieved by an ice/water bath. Organic extracts were dried over anhydrous $\mathrm{MgSO}_{4}$, and solutions were evaporated under reduced pressure with a rotatory evaporator and a bath at $40^{\circ} \mathrm{C}$.

\section{General procedure for addition of carboxylic acids dianions to $\mathrm{N}$-benzylidenebenzeneamine} The base was generated from n-butyl-lithium 1.6 $\mathrm{M}$ in hexane $(5 \mathrm{mmol})$, which was evaporated with a flow of argon and the corresponding amine $(5 \mathrm{mmol})$ dissolved in THF $(2 \mathrm{~mL})$ was added. The acid $(2.25 \mathrm{mmol})$ disolved in $2 \mathrm{~mL}$ of THF was slowly added at $-78^{\circ} \mathrm{C}$. The reaction was stirred for 0.5 hours $\left(1 \mathrm{~h}\right.$ for aromatic acids) at $0^{\circ} \mathrm{C}$ to enable dianion formation. The imine $(2.25$ or $6.75 \mathrm{mmol})$ was disolved in THF $(2 \mathrm{~mL})$ and added dropwise at $-78^{\circ} \mathrm{C}$, the reaction was stirred during 21 hours at the corresponding temperature. The reaction was quenched with water $(20$ $\mathrm{mL})$ and extracted with diethyl ether $(3 \times 15 \mathrm{~mL})$ to give non-acidic products. The aqueous phase was acidified with concentrated hydrochloric acid in an ice bath and then extracted with ethyl 
acetate $(3 \times 15 \mathrm{~mL})$. The organic phase was washed with brine and dried with $\mathrm{MgSO}_{4}$. The solvent was removed through rotary evaporation.

2,3-Diphenyl-3-(phenylamino)propanoic acid (6). Prepared from phenylacetic acid (2) (306 $\mathrm{mg}, 2.25 \mathrm{mmol})$ and $N$-benzylideneaniline (1) (1.223 g, $6.75 \mathrm{mmol})$ as a white solid; yield: 459 mg (65\%); mp 155-157 C. IR (KBr): 3500-3000 (bs); 1732; 1678; 1547; 1504; 1449; 1389; 1247; 1029; 691. ${ }^{1} \mathrm{H}$ NMR (400 MHz, $\left.\mathrm{CDCl}_{3}\right), \delta: 4.01$ (d, J=8.7 Hz, 1H, CㅂCOOH); 4.98 (d, $J$ $=8.9 \mathrm{~Hz}, 1 \mathrm{H}, \mathrm{C} \underline{\mathrm{HNH}}) ; 6.64\left(\mathrm{~d}, J=7.9 \mathrm{~Hz}, 2 \mathrm{H}, \mathrm{CH}_{\mathrm{Ar}}\right) ; 6.69\left(\mathrm{~d}, J=7.1 \mathrm{~Hz}, 1 \mathrm{H}, \mathrm{CH}_{\mathrm{Ar}}\right) ; 7.05-7.38$ $\left(\mathrm{m}, 12 \mathrm{H}, \mathrm{CH}_{\mathrm{Ar}}\right) .{ }^{13} \mathrm{C} \mathrm{NMR}\left(100 \mathrm{MHz}, \mathrm{CDCl}_{3}\right), \delta: 58.5(\underline{\mathrm{CHCOOH}}) ; 61.6(\mathrm{CHNH}) ; 114.8$ $\left(\mathrm{CH}_{\mathrm{Ar}}\right) ; 118.8\left(\mathrm{CH}_{\mathrm{Ar}}\right) ; 127.1\left(\mathrm{CH}_{\mathrm{Ar}}\right) ; 127.4\left(\mathrm{CH}_{\mathrm{Ar}}\right) ; 127.8\left(\mathrm{CH}_{\mathrm{Ar}}\right) ; 128.8\left(\mathrm{CH}_{\mathrm{Ar}}\right) ; 129.1\left(\mathrm{CH}_{\mathrm{Ar}}\right)$; $129.5\left(\mathrm{CH}_{\mathrm{Ar}}\right) ; 129.7\left(\mathrm{CH}_{\mathrm{Ar}}\right) ; 135.3\left(\mathrm{C}_{\mathrm{Ar}}\right) ; 139.5\left(\mathrm{C}_{\mathrm{Ar}}\right) ; 142.6\left(\mathrm{C}_{\mathrm{Ar}}\right) ; 177.3(\mathrm{COOH}) . \mathrm{MS}, \mathrm{m} / \mathrm{z}(\%)$ : $317\left[\mathrm{M}^{+}, 0.4 \%\right] ; 224\left[\mathrm{M}^{+}-\mathrm{PhNH}, 3.5 \%\right] ; 182\left[\mathrm{PhNHCHPh}^{+}, 100 \%\right] ; 77\left[\mathrm{C}_{6} \mathrm{H}_{5}{ }^{+}, 11.7 \%\right]$. HRMS $\mathrm{m} / \mathrm{z}[\mathrm{M}+]$ calcd for $\mathrm{C}_{21} \mathrm{H}_{19} \mathrm{NO}_{2}: 317.1416$; found: 317.1423 .

Reaction of hydrocinnamic acid (3) with $\boldsymbol{N}$-benzylideneaniline (1). From hydrocinnamic acid (3) (338 mg, $2.25 \mathrm{mmol})$ and N-benzylideneaniline (1) (1.223 g, $6.75 \mathrm{mmol})$ a brown solid was obtained; yield: $510 \mathrm{mg}(70 \%)$ as a diastereoisomeric mixture $(28: 72)$ of $\left(2 S^{*}, 3 R^{*}\right)-2-b e n z y l-3-$ phenyl-3-(phenylamino)propanoic acid (7) and $\left(2 R^{*}, 3 R^{*}\right)$-2-benzyl-3-phenyl-3(phenylamino)propanoic acid (8). Mp 125-128 C. IR (KBr): 3612; 3344; 3017; 2929; 2864; 1596; 1569; 1503; 1432; 1307. MS, m/z (\%): $331[\mathrm{M}+, 13 \%] ; 182\left[\mathrm{PhNHCHPh}^{+}, 100 \%\right] ; 91$ $\left[\mathrm{C}_{7} \mathrm{H}_{7}{ }^{+}, 8 \%\right]$. HRMS m/z $\left[\mathrm{M}^{+}\right]$calcd for $\mathrm{C}_{22} \mathrm{H}_{21} \mathrm{NO}_{2}$ : 331.1572; found: 331.1583 .

(2S*,3R*)-2-Benzyl-3-phenyl-3-(phenylamino)propanoic acid (7) minor diastereoisomer.

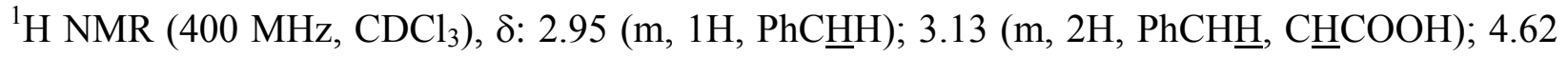
(d, $J=5.8 \mathrm{~Hz}, 1 \mathrm{H}, \mathrm{PhC} \underline{\mathrm{HNH}}) ; 6.59$ (d, $\left.J=8.5 \mathrm{~Hz}, 2 \mathrm{H}, \mathrm{CH}_{\mathrm{Ar}}\right) ; 6.72$ (t, $J=7.5 \mathrm{~Hz}, 1 \mathrm{H}, \mathrm{CH}_{\mathrm{Ar}}$ ); 7.13-7.40 (m, 12H, $\left.\mathrm{CH}_{\mathrm{Ar}}\right) .{ }^{13} \mathrm{C} \mathrm{NMR}\left(100 \mathrm{MHz}, \mathrm{CDCl}_{3}\right)$, $\delta: 33.4\left(\mathrm{PhCH}_{2}\right) ; 54.9(\underline{\mathrm{CHCOOH}})$; $58.4(\mathrm{CHNH}) ; 114.1\left(\mathrm{CH}_{\mathrm{Ar}}\right) ; 118.2\left(\mathrm{CH}_{\mathrm{Ar}}\right) ; 127.0\left(\mathrm{CH}_{\mathrm{Ar}}\right) ; 128.2\left(\mathrm{CH}_{\mathrm{Ar}}\right) ; 128.8\left(\mathrm{CH}_{\mathrm{Ar}}\right) ; 129.0$ $\left(\mathrm{CH}_{\mathrm{Ar}}\right) ; 129.2\left(\mathrm{CH}_{\mathrm{Ar}}\right) ; 129.3\left(\mathrm{CH}_{\mathrm{Ar}}\right) ; 129.6\left(\mathrm{CH}_{\mathrm{Ar}}\right) ; 138.4\left(\mathrm{C}_{\mathrm{Ar}}\right) ; 140.2\left(\mathrm{C}_{\mathrm{Ar}}\right) ; 146.9\left(\mathrm{C}_{\mathrm{Ar}}\right) ; 179.8$ $(\mathrm{COOH})$.

(2R*,3R*)-2-Benzyl-3-phenyl-3-(phenylamino)propanoic acid (8) major diastereoisomer.

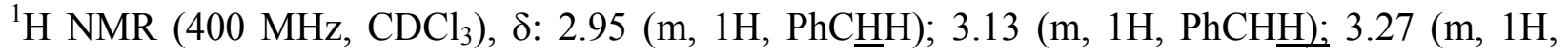

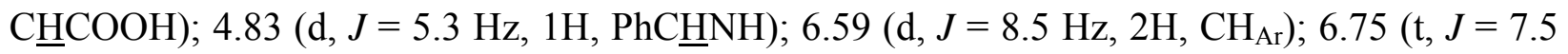
$\left.\mathrm{Hz}, 1 \mathrm{H}, \mathrm{CH}_{\mathrm{Ar}}\right) ; 7.13-7.40\left(\mathrm{~m}, 12 \mathrm{H}, \mathrm{CH}_{\mathrm{Ar}}\right) .{ }^{13} \mathrm{C} \mathrm{NMR}\left(100 \mathrm{MHz}, \mathrm{CDCl}_{3}\right), \delta: 33.4\left(\mathrm{PhCH}_{2}\right) ; 54.9$ $(\underline{\mathrm{CHCOOH}}) ; 58.4(\mathrm{CHNH}) ; 114.4\left(\mathrm{CH}_{\mathrm{Ar}}\right) ; 118.6\left(\mathrm{CH}_{\mathrm{Ar}}\right) ; 127.0\left(\mathrm{CH}_{\mathrm{Ar}}\right) ; 127.2\left(\mathrm{CH}_{\mathrm{Ar}}\right) ; 127.4$ $\left(\mathrm{CH}_{\mathrm{Ar}}\right) ; 128.8\left(\mathrm{CH}_{\mathrm{Ar}}\right) ; 129.2\left(\mathrm{CH}_{\mathrm{Ar}}\right) ; 129.3\left(\mathrm{CH}_{\mathrm{Ar}}\right) ; 129.5\left(\mathrm{CH}_{\mathrm{Ar}}\right) ; 139.2\left(\mathrm{C}_{\mathrm{Ar}}\right) ; 140.2\left(\mathrm{C}_{\mathrm{Ar}}\right) ; 146.9$ $\left(\mathrm{C}_{\mathrm{Ar}}\right) ; 178.9(\mathrm{COOH})$.

Reaction of valeric acid (4) with $\boldsymbol{N}$-benzylideneaniline (1). From valeric acid (4) (245 mg, $2.25 \mathrm{mmol})$ and N-benzylideneaniline (1) (1.223 g, $6.75 \mathrm{mmol})$ a brown solid was obtained; yield: $474 \mathrm{mg}(74 \%)$; as a diastereoisomeric mixture $(20: 80)$ of $\left(2 \boldsymbol{R}^{*}, \mathbf{3} \boldsymbol{R}^{*}\right)$-3-phenyl-3(phenylamino)-2-propylpropanoic acid (9) and $\left(2 R^{*}, 3 S^{*}\right)-3-p h e n y l-3-(p h e n y l a m i n o)-2-$ propylpropanoic acid (10). Mp $112-115^{\circ} \mathrm{C}$. IR (KBr): 3500-2400 (bs), 3409, 2959, 1705, 1602, 1505, 1454, 1263, 1158, 773. MS, m/z (\%): $283\left[\mathrm{M}^{+}, 5.6 \%\right] ; 182\left[\mathrm{PhNHCHPh}^{+}, 100 \%\right] ; 7$ $\left[\mathrm{C}_{6} \mathrm{H}_{5}{ }^{+}, 11.4 \%\right]$. HRMS $\mathrm{m} / \mathrm{z}\left[\mathrm{M}^{+}\right]$calcd for $\mathrm{C}_{18} \mathrm{H}_{21} \mathrm{NO}_{2}$ : 283.1572; found: 283.1585. 
(2R*,3R*)-3-Phenyl-3-(phenylamino)-2-propylpropanoic acid (9) minor diastereoisomer. ${ }^{1} \mathrm{H}$ NMR (400 MHz, $\mathrm{CDCl}_{3}$ ), $\delta: 0.88$ (t, $\left.J=5.4 \mathrm{~Hz}, 3 \mathrm{H}, \mathrm{CH}_{3}\right) ; 1.20-1.90\left(\mathrm{~m}, 4 \mathrm{H}, \mathrm{C}_{2} \mathrm{C}_{2}\right) ; 2.84$ (ddd, $\left.J_{1}=3.4 \mathrm{~Hz}, J_{2}=7.6 \mathrm{~Hz}, \mathrm{~J}_{3}=8.8 \mathrm{~Hz}, 1 \mathrm{H}, \mathrm{CHCOOH}\right) ; 4.58(\mathrm{~d}, J=7.6 \mathrm{~Hz}, 1 \mathrm{H}, \mathrm{CHNH}$ ), $6.58\left(\mathrm{~d}, J=7.6 \mathrm{~Hz}, 2 \mathrm{H}, \mathrm{CH}_{\mathrm{Ar}}\right) ; 6.67\left(\mathrm{t}, J=7.4 \mathrm{~Hz}, 1 \mathrm{H}, \mathrm{CH}_{\mathrm{Ar}}\right) ; 7.07-7.36\left(\mathrm{~m}, 7 \mathrm{H}, \mathrm{CH}_{\mathrm{Ar}}\right) .{ }^{13} \mathrm{C}$ NMR (100 MHz, $\left.\mathrm{CDCl}_{3}\right), \delta: 13.8\left(\mathrm{CH}_{3}\right) ; 20.7\left(\mathrm{CH}_{3} \underline{\mathrm{CH}}_{2}\right) ; 29.1\left(\underline{\left.\mathrm{CH}_{2} \mathrm{CH}\right)} ; 52.1(\underline{\mathrm{CHCOOH}}) ; 59.5\right.$ $(\mathrm{CHNH}) ; 113.8\left(\mathrm{CH}_{\mathrm{Ar}}\right) ; 117.8\left(\mathrm{CH}_{\mathrm{Ar}}\right) ; 126.8\left(\mathrm{CH}_{\mathrm{Ar}}\right) ; 127.5\left(\mathrm{CH}_{\mathrm{Ar}}\right) ; 128.7\left(\mathrm{CH}_{\mathrm{Ar}}\right) ; 129.1\left(\mathrm{CH}_{\mathrm{Ar}}\right)$; $140.9\left(\mathrm{C}_{\mathrm{Ar}}\right) ; 146.6\left(\mathrm{C}_{\mathrm{Ar}}\right) ; 180.6(\mathrm{COOH})$.

(2R*,3S*)-3-Phenyl-3-(phenylamino)-2-propylpropanoic acid (10) major diastereoisomer. ${ }^{1} \mathrm{H}$ NMR (400 MHz, $\mathrm{CDCl}_{3}$ ), $\delta: 0.89$ (t, $\left.J=7.3 \mathrm{~Hz}, 3 \mathrm{H}, \mathrm{CH}_{3}\right) ; 1.20-1.90\left(\mathrm{~m}, 4 \mathrm{H}, \mathrm{C}_{2} \mathrm{C}_{2}\right) ; 2.88$ $\left(\mathrm{ddd}, J_{1}=3.4 \mathrm{~Hz}, J_{2}=5.4 \mathrm{~Hz}, \mathrm{~J}_{3}=8.9 \mathrm{~Hz}, 1 \mathrm{H}, \mathrm{CHCOOH}\right) ; 4.72(\mathrm{~d}, J=5.5 \mathrm{~Hz}, 1 \mathrm{H}, \mathrm{CHNH})$, $6.55\left(\mathrm{~d}, J=8.5 \mathrm{~Hz}, 2 \mathrm{H}, \mathrm{CH}_{\mathrm{Ar}}\right) ; 6.69\left(\mathrm{t}, J=7.3 \mathrm{~Hz}, 1 \mathrm{H}, \mathrm{CH}_{\mathrm{Ar}}\right) ; 7.07-7.36\left(\mathrm{~m}, 7 \mathrm{H}, \mathrm{CH}_{\mathrm{Ar}}\right) .{ }^{13} \mathrm{C}$ NMR (100 MHz, $\left.\mathrm{CDCl}_{3}\right), \delta: 13.9\left(\mathrm{CH}_{3}\right) ; 21.1\left(\mathrm{CH}_{3} \underline{\mathrm{CH}}_{2}\right) ; 29.3\left(\underline{\left.\mathrm{CH}_{2} \mathrm{CH}\right)} ; 52.7(\underline{\mathrm{CHCOOH}}) ; 59.4\right.$ $(\mathrm{CHNH}) ; 113.9\left(\mathrm{CH}_{\mathrm{Ar}}\right) ; 118.0\left(\mathrm{CH}_{\mathrm{Ar}}\right) ; 126.9\left(\mathrm{CH}_{\mathrm{Ar}}\right) ; 127.6\left(\mathrm{CH}_{\mathrm{Ar}}\right) ; 128.2\left(\mathrm{CH}_{\mathrm{Ar}}\right) ; 129.4\left(\mathrm{CH}_{\mathrm{Ar}}\right)$; $140.4\left(\mathrm{C}_{\mathrm{Ar}}\right) ; 146.8\left(\mathrm{C}_{\mathrm{Ar}}\right) ; 179.8(\mathrm{COOH})$.

Reaction of crotonic acid (11) with $\boldsymbol{N}$-benzylideneaniline (1). From crotonic acid (11) (193 $\mathrm{mg}, 2.25 \mathrm{mmol}$ ) and N-benzylideneaniline (1) (408 $\mathrm{mg}, 2.25 \mathrm{mmol}$ ), a crude mixture was obtained (258 $\mathrm{mg}, 43 \%)$ as a complex mixture. It was converted into the corresponding methyl esters by reflux for $2 \mathrm{~h}$ in $\mathrm{MeOH}(20 \mathrm{~mL}) /$ sulfuric acid (2\%). Column chromatography (hexane/ethyl acetate 7:3) only allowed to isolate pure methyl 5-phenyl-5-(phenylamino)pent2-enoate (17-methyl ester) as a yellow oil. Yield $127 \mathrm{mg}$ (21\%). IR (KBr): 3391, 2952, 1725, 1659, 1602, 1501, 1436, 1318, 1200, 752. ${ }^{1} \mathrm{H} \mathrm{NMR}\left(400 \mathrm{MHz}, \mathrm{CDCl}_{3}\right), \delta: 2.62\left(\mathrm{~m}, 2 \mathrm{H}, \mathrm{CH}_{2}\right)$; $3.64\left(\mathrm{~s}, 3 \mathrm{H}, \mathrm{CH}_{3}\right) ; 4.44(\mathrm{t}, J=7.5 \mathrm{~Hz}, 1 \mathrm{H}, \mathrm{NHC} \underline{\mathrm{H}}) ; 5.85$ (d, $\left.J=15.7 \mathrm{~Hz}, 1 \mathrm{H}, \mathrm{C} \underline{\mathrm{HCOOH}}\right) ; 6.43$ $\left(\mathrm{d}, J=7.8 \mathrm{~Hz}, 2 \mathrm{H}, \mathrm{CH}_{\mathrm{Ar}}\right) ; 6.58\left(\mathrm{t}, J=7.5 \mathrm{~Hz}, 1 \mathrm{H}, \mathrm{CH}_{\mathrm{Ar}}\right) ; 6.84\left(\mathrm{~m}, 1 \mathrm{H}, \mathrm{CH}_{2} \mathrm{CH}=\right) ; 7.01(\mathrm{t}, J=7.5$ $\left.\mathrm{Hz}, 2 \mathrm{H}, \mathrm{CH}_{\mathrm{Ar}}\right) ; 7.24-7.45\left(\mathrm{~m}, 5 \mathrm{H}, \mathrm{CH}_{\mathrm{Ar}}\right) .{ }^{13} \mathrm{C} \mathrm{NMR}\left(100 \mathrm{MHz}, \mathrm{CDCl}_{3}\right) \delta: 41.1\left(\mathrm{CH}_{2}\right) ; 51.8$ $\left(\mathrm{CH}_{3}\right) ; 56.9(\mathrm{NHCH}) ; 113.5\left(\mathrm{CH}_{\mathrm{Ar}}\right) ; 117.8\left(\mathrm{CH}_{\mathrm{Ar}}\right) ; 123.8(\underline{\mathrm{CHCOOH}}) ; 126.3\left(\mathrm{CH}_{2} \underline{\mathrm{CH}}=\right) ; 127.4$ $\left(\mathrm{CH}_{\mathrm{Ar}}\right) ; 128.5\left(\mathrm{CH}_{\mathrm{Ar}}\right) ; 128.8\left(\mathrm{CH}_{\mathrm{Ar}}\right) ; 142.5\left(\mathrm{C}_{\mathrm{Ar}}\right) ; 144.8\left(\mathrm{CH}_{\mathrm{Ar}}\right) ; 146.7\left(\mathrm{C}_{\mathrm{Ar}}\right) ; 166.5(\mathrm{COOH})$.

2-(2-Aza-1,2-diphenylethyl)-3-methylbut-3-enoic acid (16). Prepared from 3-methylbut-2enoic acid (13) (225 mg, $2.25 \mathrm{mmol})$ and 1 (408 $\mathrm{mg}, 2.25 \mathrm{mmol})$ as a yellow solid; yield: 342 mg (54\%); mp $173-175^{\circ} \mathrm{C}$. IR (KBr): 3500-2500 (bs), 3400, 3028, 2924, 1702, 1603, 1504, 1265, 1177, 751. ${ }^{1} \mathrm{H}$ NMR (300 MHz, $\left.\mathrm{CDCl}_{3}\right), \delta: 1.74\left(\mathrm{~s}, 3 \mathrm{H}, \mathrm{CH}_{3}\right) ; 3.36(\mathrm{~d}, J=10.5 \mathrm{~Hz}, 1 \mathrm{H}$, $\mathrm{C} \underline{\mathrm{HCOOH}}) ; 4.59$ (d, $J=10.5 \mathrm{~Hz}, 1 \mathrm{H}, \underline{\mathrm{CHNH}}), 5.10$ (d, $J=1.5 \mathrm{~Hz}, 1 \mathrm{H}, \underline{\mathrm{CHH}}=) ; 5.12$ (br. s, $1 \mathrm{H}$, $\mathrm{CH} \underline{\mathrm{H}}=) ; 6.45\left(\mathrm{~d}, J=7.5 \mathrm{~Hz}, 2 \mathrm{H}, \mathrm{CH}_{\mathrm{Ar}}\right) ; 6.59$ (t, $\left.J=7.5 \mathrm{~Hz}, 1 \mathrm{H}, \mathrm{CH}_{\mathrm{Ar}}\right) ; 6.99(\mathrm{t}, J=7.5 \mathrm{~Hz}, 2 \mathrm{H}$, $\left.\mathrm{CH}_{\mathrm{Ar}}\right) ; 7.16-7.24\left(\mathrm{~m}, 3 \mathrm{H}, \mathrm{CH}_{\mathrm{Ar}}\right) ; 7.35\left(\mathrm{dd}, J_{1}=1.8 \mathrm{~Hz}, J_{2}=8.1 \mathrm{~Hz}, 2 \mathrm{H}, \mathrm{CH}_{\mathrm{Ar}}\right) .{ }^{13} \mathrm{C} \mathrm{NMR}(75$ $\left.\mathrm{MHz}, \mathrm{CDCl}_{3}\right), \delta: 19.2\left(\mathrm{CH}_{3}\right), 56.9(\mathrm{CHNH}), 62.2(\underline{\mathrm{CHCOOH}}) ; 114.2\left(\mathrm{CH}_{\mathrm{Ar}}\right) ; 118.5\left(\mathrm{CH}_{\mathrm{Ar}}\right)$; $119.0\left(\mathrm{CH}_{\mathrm{Ar}}\right) ; 127.7\left(\mathrm{CH}_{\mathrm{Ar}}\right) ; 128.1\left(\mathrm{CH}_{\mathrm{Ar}}\right) ; 129.0\left(\mathrm{CH}_{\mathrm{Ar}}\right) ; 129.5\left(\mathrm{CH}_{\mathrm{Ar}}\right) ; 140.4\left(\mathrm{CH}_{3} \underline{\mathrm{C}}=\right) ; 141.5$ $\left(\mathrm{C}_{\mathrm{Ar}}\right) ; 147.0\left(\mathrm{C}_{\mathrm{Ar}}\right) ; 175.4(\mathrm{COOH}) . \mathrm{MS}, \mathrm{m} / \mathrm{z}(\%): 281[\mathrm{M}+, 1 \%] ; 182\left[\mathrm{C}_{13} \mathrm{H}_{13} \mathrm{~N}+, 100 \%\right], 104$ $\left[\mathrm{C}_{7} \mathrm{H}_{6} \mathrm{~N}+, 10 \%\right] ; 91\left[\mathrm{C}_{7} \mathrm{H}_{7}^{+}, 2 \%\right] ; 77\left[\mathrm{C}_{6} \mathrm{H}_{5}^{+}, 22 \%\right]$. HRMS, m/z $\left[\mathrm{M}^{+}\right]$calcd for $\mathrm{C}_{18} \mathrm{H}_{19} \mathrm{NO}_{2}$ : 281.1416; found: 281.1417.

(E)-2-Methyl-5-phenyl-5-(phenylamino)pent-2-enoic acid (18). Prepared from (E)-2methylbut-2-enoic acid (12) (225 mg, $2.25 \mathrm{mmol}$ ) and $\mathbf{1}$ (408 mg, $2.25 \mathrm{mmol}$ ) as a yellow solid; 
yield: $481 \mathrm{mg}(76 \%)$; mp 133-135 ${ }^{\circ} \mathrm{C}$. IR (KBr): 3405, 3025, 1685, 1601, 1504, 1452, 1421, 1283, 1078, 1027, 933, 870. ${ }^{1} \mathrm{H}$ NMR (400 MHz, $\left.\mathrm{CDCl}_{3}\right), \delta: 1.89\left(\mathrm{~s}, 3 \mathrm{H}, \mathrm{CH}_{3}\right) ; 2.81$ (t, $J=6.7$ $\left.\mathrm{Hz}, 2 \mathrm{H}, \mathrm{CH}_{2}\right) ; 4.62(\mathrm{t}, J=6.6 \mathrm{~Hz}, 1 \mathrm{H}, \mathrm{PhCH}) ; 6.66\left(\mathrm{~d}, J=8.2 \mathrm{~Hz}, 2 \mathrm{H}, \mathrm{CH}_{\mathrm{Ar}}\right) ; 6.79(\mathrm{t}, J=7.3$ $\left.\mathrm{Hz}, 1 \mathrm{H}, \mathrm{CH}_{\mathrm{Ar}}\right) ; 7.07(\mathrm{t}, J=6.6 \mathrm{~Hz}, 1 \mathrm{H}, \mathrm{CH}=\mathrm{C}) ; 7.21\left(\mathrm{t}, J=7.6 \mathrm{~Hz}, 2 \mathrm{H}, \mathrm{CH}_{\mathrm{Ar}}\right) ; 7.34(\mathrm{~m}, 1 \mathrm{H}$, $\left.\mathrm{CH}_{\mathrm{Ar}}\right) ; 7.42\left(\mathrm{~m}, 2 \mathrm{H}, \mathrm{CH}_{\mathrm{Ar}}\right) ; 7.45\left(\mathrm{~m}, 2 \mathrm{H}, \mathrm{CH}_{\mathrm{Ar}}\right) .{ }^{13} \mathrm{C} \mathrm{NMR}\left(100 \mathrm{MHz}, \mathrm{CDCl}_{3}\right), \delta: 12.5\left(\mathrm{CH}_{3}\right)$; $38.1\left(\mathrm{CH}_{2}\right) ; 58.3(\mathrm{PhCH}) ; 114.1\left(\mathrm{CH}_{\mathrm{Ar}}\right) ; 118.3\left(\mathrm{CH}_{\mathrm{Ar}}\right) ; 125.0\left(\mathrm{CH}_{\mathrm{Ar}}\right) ; 126.2\left(\mathrm{CH}_{\mathrm{Ar}}\right) ; 127.0$ $\left(\mathrm{CH}_{\mathrm{Ar}}\right) ; 130.1\left(\mathrm{CH}_{3}-\underline{\mathrm{C}}=\right) ; 138.3\left(\mathrm{C}_{\mathrm{Ar}}\right) ; 141.0(\underline{\mathrm{CH}}=\mathrm{C}) ; 142.4\left(\mathrm{C}_{\mathrm{Ar}}\right) ; 171.2(\mathrm{COOH}) . \mathrm{MS}, \mathrm{m} / \mathrm{z}(\%)$ : $281\left[\mathrm{M}^{+}, 1 \%\right] ; 263\left[\mathrm{M}^{+}-\mathrm{H}_{2} \mathrm{O}, 1 \%\right] ; 182\left[\mathrm{C}_{13} \mathrm{H}_{12} \mathrm{~N}^{+}, 100\right] ; 104\left[\mathrm{C}_{7} \mathrm{H}_{6} \mathrm{~N}^{+}, 7 \%\right] ; 91\left[\mathrm{C}_{7} \mathrm{H}_{7}^{+}, 1 \%\right] ; 77$ $\left[\mathrm{C}_{6} \mathrm{H}_{5}{ }^{+}, 10 \%\right]$. HRMS, $\mathrm{m} / \mathrm{z}\left[\mathrm{M}^{+}\right]$calcd for $\mathrm{C}_{18} \mathrm{H}_{19} \mathrm{NO}_{2}$ : 281.1416; found: 281.1396.

From the same reagents, using lithium diethylamide as base and $21 \mathrm{~h}$ at room temperature as reaction conditions, a yelow solid was obtained $(296 \mathrm{mg}, 70 \%)$ that was identified as (E,E)-2methyl-5-phenyl-2,4-pentadienoic acid. ${ }^{18}$

2-(3-Aza-2,3-diphenylpropyl)nicotinic acid (29). Prepared from 2-methylnicotinic acid (24) (309 mg, $2.25 \mathrm{mmol})$ and N-benzylideneaniline (1) (1.223 g, $6.75 \mathrm{mmol})$ as yellow oil; yield: 544 mg (76\%). IR (KBr): 3500-2500 (bs); 3029; 2961; 1716; 1602; 1505; 1455; 1381; 1209; 749. ${ }^{1} \mathrm{H}$ NMR $\left(400 \mathrm{MHz}, \mathrm{CDCl}_{3}\right), \delta: 3.42\left(\mathrm{dd}, J_{1}=8.8 \mathrm{~Hz}, J_{2}=13.6 \mathrm{~Hz}, 1 \mathrm{H}, \mathrm{C} \underline{\mathrm{HH}}\right) ; 3.50\left(\mathrm{dd}, J_{1}\right.$ $\left.=5.6 \mathrm{~Hz}, J_{2}=13.6 \mathrm{~Hz}, 1 \mathrm{H}, \mathrm{CH} \underline{\mathrm{H}}\right) ; 4.82\left(\mathrm{dd}, J_{1}=5.7 \mathrm{~Hz}, J_{2}=8.5 \mathrm{~Hz}, 1 \mathrm{H}, \mathrm{CH}_{2} \mathrm{C} \underline{\mathrm{H}}\right) ; 6.33(\mathrm{~m}, 3 \mathrm{H}$, $\left.\mathrm{CH}_{\mathrm{Ar}}\right) ; 6.84\left(\mathrm{t}, J=7.7 \mathrm{~Hz}, 2 \mathrm{H}, \mathrm{CH}_{\mathrm{Ar}}\right) ; 7.07\left(\mathrm{t}, J=7.3 \mathrm{~Hz}, 1 \mathrm{H}, \mathrm{CH}_{\mathrm{Ar}}\right) ; 7.17(\mathrm{t}, J=7.3 \mathrm{~Hz}, 2 \mathrm{H}$, $\left.\mathrm{CH}_{\mathrm{Ar}}\right) ; 7.26\left(\mathrm{~m}, 3 \mathrm{H}, \mathrm{CH}_{\mathrm{Ar}}\right) ; 8.04\left(\mathrm{dd}, J_{1}=1.8 \mathrm{~Hz}, J_{2}=7.9 \mathrm{~Hz}, 1 \mathrm{H}, \mathrm{CH}_{\mathrm{Ar}}\right) ; 8.58\left(\mathrm{dd}, J_{1}=1.8 \mathrm{~Hz}\right.$, $\left.J_{2}=4.8 \mathrm{~Hz}, 1 \mathrm{H}, \mathrm{CH}_{\mathrm{Ar}}\right) .{ }^{13} \mathrm{C} \mathrm{NMR}\left(100 \mathrm{MHz}, \mathrm{CDCl}_{3}\right), \delta: 44.0\left(\mathrm{CH}_{2}\right) ; 56.9\left(\mathrm{CH}_{2} \mathrm{CH}\right) ; 112.3$ $\left(\mathrm{CH}_{\mathrm{Ar}}\right) ; 116.0\left(\mathrm{CH}_{\mathrm{Ar}}\right) ; 121.6\left(\mathrm{CH}_{\mathrm{Ar}}\right) ; 126.3\left(\mathrm{C}_{\mathrm{Ar}}\right) ; 126.6\left(\mathrm{CH}_{\mathrm{Ar}}\right) ; 126.7\left(\mathrm{CH}_{\mathrm{Ar}}\right) ; 128.2\left(\mathrm{CH}_{\mathrm{Ar}}\right)$; $128.6\left(\mathrm{CH}_{\mathrm{Ar}}\right) ; 138.2\left(\mathrm{CH}_{\mathrm{Ar}}\right) ; 144.1\left(\mathrm{C}_{\mathrm{Ar}}\right) ; 147.7\left(\mathrm{C}_{\mathrm{Ar}}\right) ; 151.3\left(\mathrm{C}_{\mathrm{Ar}}\right) ; 158.8\left(\mathrm{C}_{\mathrm{Ar}}\right) ; 168.1(\mathrm{COOH})$. MS, m/z (\%): $318\left[\mathrm{M}^{+}, 4.8 \%\right] ; 300\left[\mathrm{M}^{+}-\mathrm{H}_{2} \mathrm{O}, 14.1 \%\right] ; 182\left[\mathrm{PhNHCHPh}^{+}, 100 \%\right] ; 77\left[\mathrm{C}_{6} \mathrm{H}_{5}^{+}\right.$, 13.6\%]. HRMS, $m / z$ [M ${ }^{+}$] calcd for $\mathrm{C}_{20} \mathrm{H}_{18} \mathrm{~N}_{2} \mathrm{O}_{2}$ : 318.1368; found: 318.1361 .

After 1 day at room temperature, 29 is spontaneously converted, in quantitative yield, into 7,8Dihydro-6,7-diphenyl-1,6-naphthyridin-5(6H)-one (34). Yellow oil. IR (KBr): 3161, 2942, 1670, 1565, 1465, 1418, 1337, 1311, 1029, 659. ${ }^{1} \mathrm{H}$ NMR $\left(300 \mathrm{MHz}, \mathrm{CDCl}_{3}\right), \delta: 3.36\left(\mathrm{dd}, J_{1}=\right.$ $\left.16.5 \mathrm{~Hz}, J_{2}=2.4 \mathrm{~Hz}, 1 \mathrm{H}, \mathrm{C} \underline{\mathrm{HH}}\right) ; 3.92\left(\mathrm{dd}, J_{1}=16.5 \mathrm{~Hz}, J_{2}=6.6 \mathrm{~Hz}, 1 \mathrm{H}, \mathrm{CH} \underline{\mathrm{H}}\right) ; 5.25\left(\mathrm{dd}, J_{1}=\right.$ $\left.6.6 \mathrm{~Hz}, J_{2}=2.4 \mathrm{~Hz}, 1 \mathrm{H}, \mathrm{PhC} \underline{\mathrm{H}}\right) ; 7.09-7.28(\mathrm{~m}, 11 \mathrm{H}, \mathrm{CHAr}), 8.38$ (dd, $J_{1}=7.8 \mathrm{~Hz}, J_{2}=1.8 \mathrm{~Hz}$, $\left.1 \mathrm{H}, \mathrm{CH}_{\mathrm{Ar}}\right) ; 8.49\left(\mathrm{dd}, J_{1}=5.1 \mathrm{~Hz}, J_{2}=1.8 \mathrm{~Hz}, 1 \mathrm{H}, \mathrm{CH}_{\mathrm{Ar}}\right) .{ }^{13} \mathrm{C} \mathrm{NMR}\left(75 \mathrm{MHz}, \mathrm{CDCl}_{3}\right), \delta: 39.3$ $\left(\mathrm{CH}_{2}\right) ; 62.6(\mathrm{PhCH}) ; 122.7\left(\mathrm{CH}_{\mathrm{Ar}}\right) ; 125.5\left(\mathrm{CH}_{\mathrm{Ar}}\right) ; 125.9\left(\mathrm{CH}_{\mathrm{Ar}}\right) ; 126.2\left(\mathrm{CH}_{\mathrm{Ar}}\right) ; 126.8\left(\mathrm{CH}_{\mathrm{Ar}}\right)$; $127.7\left(\mathrm{CH}_{\mathrm{Ar}}\right) ; 128.7\left(\mathrm{CH}_{\mathrm{Ar}}\right) ; 128.9\left(\mathrm{CH}_{\mathrm{Ar}}\right) ; 135.9\left(\mathrm{CH}_{\mathrm{Ar}}\right) ; 139.9\left(\mathrm{C}_{\mathrm{Ar}}\right) ; 141.9\left(\mathrm{C}_{\mathrm{Ar}}\right) ; 152.6\left(\mathrm{C}_{\mathrm{Ar}}\right)$; $155.6\left(\mathrm{C}_{\mathrm{Ar}}\right) ; 163.5(\mathrm{C}=\mathrm{O})$. MS, $\mathrm{m} / \mathrm{z}(\%): 300\left[\mathrm{M}^{+}, 100 \%\right] ; 223\left[\mathrm{M}^{+}-\mathrm{C}_{6} \mathrm{H}_{5}, 68 \%\right] ; 180\left[\mathrm{C}_{13} \mathrm{H}_{10} \mathrm{~N}^{+}\right.$, $44 \%] ; 119\left[\mathrm{C}_{7} \mathrm{H}_{5} \mathrm{NO}^{+}, 46 \%\right]$. HRMS m/z [M $]$ calcd for $\mathrm{C}_{20} \mathrm{H}_{16} \mathrm{~N}_{2} \mathrm{O}: 300.1263$; found: 300.1269 .

3-(3-Aza-2,3-diphenylpropyl)picolinic acid (30). Prepared from 3-methylpicolinic acid (25) (309 $\mathrm{mg}, 2.25 \mathrm{mmol})$ and N-benzylideneaniline (1) (1.223 g, $6.75 \mathrm{mmol})$ as yellow oil; yield: $193 \mathrm{mg}$ (27\%). IR (KBr): 3296; 3057; 3028; 2932;1755; 1663; 1601; 1497; 1452; 1355. ${ }^{1} \mathrm{H}$ NMR (300 MHz, $\left.\mathrm{CDCl}_{3}\right), \delta: 3.44\left(\mathrm{dd}, J_{1}=3.4 \mathrm{~Hz}, J_{2}=13 \mathrm{~Hz}, 1 \mathrm{H}, \mathrm{CH} H\right) ; 3.87$ (dd, $J_{1}=9.8 \mathrm{~Hz}$, $\left.J_{2}=12.7 \mathrm{~Hz}, 1 \mathrm{H}, \mathrm{CH} \underline{\mathrm{H}}\right) ; 4.70\left(\mathrm{dd}, J_{1}=4.2 \mathrm{~Hz}, J_{2}=9.6 \mathrm{~Hz}, 1 \mathrm{H}, \mathrm{CH}_{2} \mathrm{C} \underline{\mathrm{H}}\right) ; 6.44(\mathrm{~d}, J=8.1 \mathrm{~Hz}, 2 \mathrm{H}$, $\left.\mathrm{CH}_{\mathrm{Ar}}\right) ; 6.55\left(\mathrm{t}, J=7.1 \mathrm{~Hz}, 1 \mathrm{H}, \mathrm{CH}_{\mathrm{Ar}}\right) ; 7.00\left(\mathrm{t}, J=7.4 \mathrm{~Hz}, 2 \mathrm{H}, \mathrm{CH}_{\mathrm{Ar}}\right) ; 7.23\left(\mathrm{~m}, 3 \mathrm{H}, \mathrm{CH}_{\mathrm{Ar}}\right) ; 7.31(\mathrm{t}$, 
$\left.J=7.8 \mathrm{~Hz}, 1 \mathrm{H}, \mathrm{CH}_{\mathrm{Ar}}\right) ; 7.43\left(\mathrm{~d}, J=7.8 \mathrm{~Hz}, 1 \mathrm{H}, \mathrm{CH}_{\mathrm{Ar}}\right) ; 7.67\left(\mathrm{~d}, J=7.8 \mathrm{~Hz}, 2 \mathrm{H}, \mathrm{CH}_{\mathrm{Ar}}\right) ; 8.54(\mathrm{~s}$, bs, 1H, $\left.\mathrm{CH}_{\mathrm{Ar}}\right) .{ }^{13} \mathrm{C} \mathrm{NMR}\left(100 \mathrm{MHz}, \mathrm{CDCl}_{3}\right), \delta: 41.2\left(\mathrm{CH}_{2}\right) ; 60.4\left(\mathrm{CH}_{2} \mathrm{CH}\right) ; 113.4\left(\mathrm{CH}_{\mathrm{Ar}}\right) ; 117.2$ $\left(\mathrm{CH}_{\mathrm{Ar}}\right) ; 125.9\left(\mathrm{CH}_{\mathrm{Ar}}\right) ; 126.4\left(\mathrm{C}_{\mathrm{Ar}}\right) ; 126.8\left(\mathrm{CH}_{\mathrm{Ar}}\right) ; 127.3\left(\mathrm{CH}_{\mathrm{Ar}}\right) ; 128.4\left(\mathrm{CH}_{\mathrm{Ar}}\right) ; 128.7\left(\mathrm{CH}_{\mathrm{Ar}}\right)$; $129.9\left(\mathrm{CH}_{\mathrm{Ar}}\right) ; 142.5\left(\mathrm{CH}_{\mathrm{Ar}}\right) ; 143.1\left(\mathrm{C}_{\mathrm{Ar}}\right) ; 145.4\left(\mathrm{C}_{\mathrm{Ar}}\right) ; 147.0\left(\mathrm{C}_{\mathrm{Ar}}\right) ; 165.3(\mathrm{COOH}) . \mathrm{MS}, \mathrm{m} / \mathrm{z}(\%)$ : $318\left[\mathrm{M}^{+}, 5 \%\right] ; 300\left[\mathrm{M}^{+}-\mathrm{H}_{2} \mathrm{O}, 14 \%\right] ; 182\left[\mathrm{PhNHCHPh}^{+}, 100 \%\right] ; 77\left[\mathrm{C}_{6} \mathrm{H}_{5}{ }^{+}, 24 \%\right] . \mathrm{HRMS}, \mathrm{m} / \mathrm{z}$ $\left[\mathrm{M}^{+}\right]$calcd for $\mathrm{C}_{20} \mathrm{H}_{18} \mathrm{~N}_{2} \mathrm{O}_{2}: 318.1368$; found: 318.1360 .

After 1 day at room temperature, 30 is spontaneously converted, in quantitative yield, into 6,7dihydro-6,7-diphenyl-1,7-naphthyridin-8(5H)-one (35). Yellow oil. IR (KBr): 3170, 2951, 1696, 1576, 1496, 1418, 1339, 1312, 1198, 668. ${ }^{1} \mathrm{H} \mathrm{NMR}\left(400 \mathrm{MHz}, \mathrm{CDCl}_{3}\right), \delta: 3.18\left(\mathrm{dd}, J_{1}=\right.$ $\left.15.9 \mathrm{~Hz}, J_{2}=2.2 \mathrm{~Hz}, 1 \mathrm{H}, \mathrm{CHH}\right) ; 3.97\left(\mathrm{dd}, J_{1}=15.9 \mathrm{~Hz}, J_{2}=6.3 \mathrm{~Hz}, 1 \mathrm{H}, \mathrm{CH} \underline{\mathrm{H}}\right) ; 5.30\left(\mathrm{dd}, J_{1}=\right.$ $\left.6.1 \mathrm{~Hz}, J_{2}=2.0 \mathrm{~Hz}, 1 \mathrm{H}, \mathrm{PhC} \underline{\mathrm{H}}\right) ; 7.19-7.61(\mathrm{~m}, 12 \mathrm{H}, \mathrm{CHAr}), 8.73\left(\mathrm{~s}, 1 \mathrm{H}, \mathrm{CH}_{\mathrm{Ar}}\right) .{ }^{13} \mathrm{C} \mathrm{NMR}(100$ $\left.\mathrm{MHz}, \mathrm{CDCl}_{3}\right), \delta: 36.0\left(\mathrm{CH}_{2}\right) ; 62.8(\mathrm{PhCH}) ; 125.9\left(\mathrm{CH}_{\mathrm{Ar}}\right) ; 126.5\left(\mathrm{CH}_{\mathrm{Ar}}\right) ; 126.8\left(\mathrm{CH}_{\mathrm{Ar}}\right) ; 127.8$ $\left(\mathrm{CH}_{\mathrm{Ar}}\right) ; 128.7\left(\mathrm{CH}_{\mathrm{Ar}}\right) ; 128.8\left(\mathrm{CH}_{\mathrm{Ar}}\right) ; 131.6\left(\mathrm{CH}_{\mathrm{Ar}}\right) ; 136.5\left(\mathrm{C}_{\mathrm{Ar}}\right) ; 139.7\left(\mathrm{CH}_{\mathrm{Ar}}\right) ; 142.2\left(\mathrm{C}_{\mathrm{Ar}}\right) ; 146.7$ $\left(\mathrm{C}_{\mathrm{Ar}}\right) ; 149.3\left(\mathrm{C}_{\mathrm{Ar}}\right) ; 162.6(\mathrm{C}=\mathrm{O})$. MS, m/z (\%): $300\left[\mathrm{M}^{+}, 100 \%\right] ; 223\left[\mathrm{M}^{+}-\mathrm{C}_{6} \mathrm{H}_{5}, 18 \%\right] ; 180$ $\left[\mathrm{C}_{13} \mathrm{H}_{10} \mathrm{~N}^{+}, 18 \%\right] ; 119\left[\mathrm{C}_{7} \mathrm{H}_{5} \mathrm{NO}^{+}, 5 \%\right], 91\left[\mathrm{C}_{7} \mathrm{H}_{7}^{+}, 7 \%\right]$. HRMS m/z $\left[\mathrm{M}^{+}\right]$calcd for $\mathrm{C}_{20} \mathrm{H}_{16} \mathrm{~N}_{2} \mathrm{O}$ : 300.1263; found: 300.1274 .

3-(3-Aza-2,3-diphenylpropyl)thiophene-2-carboxylic acid (31). Prepared from 3methylthiophene-2-carboxylic acid (26) (320mg, $2.25 \mathrm{mmol}$ ) and N-benzylideneaniline (1) (1.223 g, $6.75 \mathrm{mmol})$ as yellow oil; yield: $276 \mathrm{mg}$ (38\%). IR (KBr): 3100-2950 (bs); 1679; 1596; $1570 ; 1501 ; 1453 ; 1323 ; 696 .{ }^{1} \mathrm{H}$ NMR $\left(400 \mathrm{MHz}, \mathrm{CDCl}_{3}\right), \delta: 3.41$ (dd, $J_{1}=4.5 \mathrm{~Hz}, J_{2}=14 \mathrm{~Hz}$, $1 \mathrm{H}, \mathrm{C} \underline{\mathrm{HH}}) ; 3.61\left(\mathrm{dd}, J_{1}=9.4 \mathrm{~Hz}, J_{2}=14 \mathrm{~Hz}, 1 \mathrm{H}, \mathrm{CH} \underline{\mathrm{H}}\right) ; 4.68\left(\mathrm{dd}, J_{1}=4.6 \mathrm{~Hz}, J_{2}=8.7 \mathrm{~Hz}, 1 \mathrm{H}\right.$, $\mathrm{C} \underline{\mathrm{HNH}}) ; 6.63\left(\mathrm{~d}, J=8.6 \mathrm{~Hz}, 2 \mathrm{H}, \mathrm{CH}_{\mathrm{Ar}}\right) ; 6.76\left(\mathrm{t}, J=7.3 \mathrm{~Hz}, 1 \mathrm{H}, \mathrm{CH}_{\mathrm{Ar}}\right) ; 6.86(\mathrm{~d}, J=4.9 \mathrm{~Hz}, 1 \mathrm{H}$, $\mathrm{SCH}=\mathrm{C} \underline{\mathrm{H}}) ; 7.05\left(\mathrm{t}, J=7.5 \mathrm{~Hz}, 2 \mathrm{H}, \mathrm{CH}_{\mathrm{Ar}}\right) ; 7.23-7.43\left(\mathrm{~m}, 5 \mathrm{H}, \mathrm{CH}_{\mathrm{Ar}}\right) ; 7.47(\mathrm{~d}, J=5.0 \mathrm{~Hz}, 1 \mathrm{H}$, $\mathrm{SCH}=\mathrm{CH}) .{ }^{13} \mathrm{C}$ NMR $\left(100 \mathrm{MHz}, \mathrm{CDCl}_{3}\right), \delta: 38.5\left(\mathrm{CH}_{2}\right) ; 59.4\left(\mathrm{CH}_{2} \mathrm{CH}\right) ; 113.4\left(\mathrm{CH}_{\mathrm{Ar}}\right) ; 117.3$ $\left(\mathrm{CH}_{\mathrm{Ar}}\right) ; 126.4\left(\mathrm{CH}_{\mathrm{Ar}}\right) ; 127.2\left(\mathrm{CH}_{\mathrm{Ar}}\right) ; 128.7\left(\mathrm{CH}_{\mathrm{Ar}}\right) ; 129.3\left(\mathrm{CH}_{\mathrm{Ar}}\right) ; 129.7\left(\mathrm{CH}_{\mathrm{Ar}}\right) ; 132.2\left(\mathrm{CH}_{\mathrm{Ar}}\right)$; $143.3\left(\mathrm{C}_{\mathrm{Ar}}\right) ; 147.2\left(\mathrm{C}_{\mathrm{Ar}}\right) ; 148.3\left(\mathrm{C}_{\mathrm{Ar}}\right) ; 154.8\left(\mathrm{CH}_{\mathrm{Ar}}\right) ; 168.4(\mathrm{COOH}) . \mathrm{MS}, \mathrm{m} / \mathrm{z}(\%): 323\left[\mathrm{M}^{+}\right.$, 8.7\%]; $305\left[\mathrm{M}^{+}-\mathrm{H}_{2} \mathrm{O}, 2.0 \%\right] ; 321\left[\mathrm{M}^{+}-\mathrm{PhNH}, 63.1 \%\right] ; 182\left[\mathrm{PhNHCHPh}^{+}, 100 \%\right] ; 77\left[\mathrm{C}_{6} \mathrm{H}_{5}^{+}\right.$, $30.9 \%$.

HRMS: $m / z\left[\mathrm{M}^{+}\right]$calcd for $\mathrm{C}_{19} \mathrm{H}_{17} \mathrm{NO}_{2} \mathrm{~S}: 323.0980$; found: 323.0989 .

2-(3-Aza-2,3-diphenylpropyl)-5-methyl-furoic acid (32). Prepared from 2,5-dimethyl-3-furoic acid (27) (315 mg, $2.25 \mathrm{mmol}$ ) and N-benzylideneaniline (1) (1.223 g, $6.75 \mathrm{mmol})$ as a yellow oil; yield: 376 mg (52\%). IR (KBr): 3500-2500 (bs); 3055; 3027; 1681; 1601; 1582; 1504; 1453; 1072; 750. ${ }^{1} \mathrm{H} \mathrm{NMR}\left(300 \mathrm{MHz}, \mathrm{CDCl}_{3}\right) \delta: 2.24\left(\mathrm{~s}, 3 \mathrm{H}, \mathrm{CH}_{3}\right) ; 3.34\left(\mathrm{dd}, J_{1}=14.4 \mathrm{~Hz}, J_{2}=4.5 \mathrm{~Hz}\right.$, $1 \mathrm{H}, \mathrm{C} \underline{\mathrm{HH}}) ; 3.51\left(\mathrm{dd}, J_{1}=14.7 \mathrm{~Hz}, J_{2}=9.9 \mathrm{~Hz}, 1 \mathrm{H}, \mathrm{CH} \underline{\mathrm{H}}\right) ; 4.73\left(\mathrm{dd}, J_{1}=4.5 \mathrm{~Hz}, J_{2}=9.6 \mathrm{~Hz}, 1 \mathrm{H}\right.$, C파); 6.25 (s, 1H, $\left.\mathrm{CH}_{3} \mathrm{CC} \underline{\mathrm{H}}\right) ; 6.50\left(\mathrm{~d}, J=7.8 \mathrm{~Hz}, 2 \mathrm{H}, \mathrm{CH}_{\mathrm{Ar}}\right) ; 6.63$ (t, J=7.5Hz, $1 \mathrm{H}, \mathrm{CH}_{\mathrm{Ar}}$ ); $7.05\left(\mathrm{t}, J=8.4 \mathrm{~Hz}, 2 \mathrm{H}, \mathrm{CH}_{\mathrm{Ar}}\right) ; 7.24-7.45\left(\mathrm{~m}, 5 \mathrm{H}, \mathrm{CH}_{\mathrm{Ar}}\right) .{ }^{13} \mathrm{C} \mathrm{NMR}\left(75 \mathrm{MHz}, \mathrm{CDCl}_{3}\right), \delta: 13.3$ $\left(\mathrm{CH}_{3}\right) ; 36.6\left(\mathrm{CH}_{2}\right) ; 58.4\left(\mathrm{CH}_{2} \underline{\mathrm{CH}}\right) ; 106.5(\underline{\mathrm{CHCCOOH}}) ; 113.6\left(\mathrm{CH}_{\mathrm{Ar}}\right) ; 115.0\left(\mathrm{CH}_{\mathrm{Ar}}\right) ; 117.7$ $(\mathrm{CH} \underline{\mathrm{COOH}}) ; 121.0\left(\mathrm{CH}_{\mathrm{Ar}}\right) ; 126.3\left(\mathrm{CH}_{\mathrm{Ar}}\right) ; 128.7\left(\mathrm{CH}_{\mathrm{Ar}}\right) ; 129.0\left(\mathrm{CH}_{\mathrm{Ar}}\right) ; 144.7\left(\mathrm{C}_{\mathrm{Ar}}\right) ; 146.2$ $\left(\mathrm{C}_{\mathrm{Ar}}\right) ; 151.4\left(\mathrm{CH}_{3} \underline{\mathrm{C}}\right) ; 169.7(\mathrm{COOH}) . \mathrm{MS} m / \mathrm{z}(\%): 321\left[\mathrm{M}^{+}, 0.7 \%\right] ; 303\left[\mathrm{M}^{+}-\mathrm{H}_{2} \mathrm{O}, 0.6 \%\right] ; 182$ 
$\left[\mathrm{PhNHCHPh}^{+}, 100 \%\right] ; 140\left[\mathrm{C}_{7} \mathrm{H}_{8} \mathrm{O}_{3}{ }^{+}, 3.4 \%\right] ; 77\left[\mathrm{C}_{6} \mathrm{H}_{5}{ }^{+}, 14.7 \%\right] . \mathrm{HRMS}, \mathrm{m} / \mathrm{z}\left[\mathrm{M}^{+}\right]$calcd for $\mathrm{C}_{20} \mathrm{H}_{19} \mathrm{NO}_{3}$ : 321.1365 ; found: 321.1359 .

3,4-Dihydro-2,3-diphenylisoquinolin-1(2H)-one (33). Prepared from o-toluic acid (23) (305 $\mathrm{mg}, 2.25 \mathrm{mmol})$ and N-benzylideneaniline (1) (1.223 g, $6.75 \mathrm{mmol})$ as a white solid; yield: 323 mg (48\%); Mp 108-109 C. IR (KBr): 3159, 3128, 1669, 1603, 1489, 1457, 1418, 1338, 1198 , 695. ${ }^{1} \mathrm{H}$ NMR (400 MHz, $\left.\mathrm{CDCl}_{3}\right), \delta: 3.17\left(\mathrm{dd}, J_{1}=15.8 \mathrm{~Hz}, J_{2}=2.7 \mathrm{~Hz}, 1 \mathrm{H}, \mathrm{CH} H\right) ; 3.91\left(\mathrm{dd}, J_{1}\right.$ $\left.=15.7 \mathrm{~Hz}, J_{2}=6.5 \mathrm{~Hz}, 1 \mathrm{H}, \mathrm{CH} \underline{\mathrm{H}}\right) ; 5.24\left(\mathrm{dd}, J_{1}=6.3 \mathrm{~Hz}, J_{2}=2.7 \mathrm{~Hz}, 1 \mathrm{H}, \mathrm{PhCH}\right) ; 7.19-7.39(\mathrm{~m}$, 13H, CHAr), $8.27\left(\mathrm{~d}, J=6.5 \mathrm{~Hz}, 1 \mathrm{H}, \mathrm{CH}_{\mathrm{Ar}}\right) .{ }^{13} \mathrm{C} \mathrm{NMR}\left(100 \mathrm{MHz}, \mathrm{CDCl}_{3}\right), \delta: 36.7\left(\mathrm{CH}_{2}\right) ; 63.4$ $(\mathrm{CHN}) ; 126.2\left(\mathrm{CH}_{\mathrm{Ar}}\right) ; 126.5\left(\mathrm{CH}_{\mathrm{Ar}}\right) ; 126.6\left(\mathrm{CH}_{\mathrm{Ar}}\right) ; 127.6\left(\mathrm{CH}_{\mathrm{Ar}}\right) ; 127.7\left(\mathrm{CH}_{\mathrm{Ar}}\right) ; 128.4\left(\mathrm{CH}_{\mathrm{Ar}}\right)$; $128.6\left(\mathrm{CH}_{\mathrm{Ar}}\right) ; 128.9\left(\mathrm{CH}_{\mathrm{Ar}}\right) ; 132.4\left(\mathrm{CH}_{\mathrm{Ar}}\right) ; 132.8\left(\mathrm{CH}_{\mathrm{Ar}}\right) ; 135.4\left(\mathrm{C}_{\mathrm{Ar}}\right) ; 140.5\left(\mathrm{C}_{\mathrm{Ar}}\right) ; 141.2\left(\mathrm{C}_{\mathrm{Ar}}\right)$; $142.6\left(\mathrm{C}_{\mathrm{Ar}}\right) ; 164.6(\mathrm{C}=\mathrm{O}) . \mathrm{MS}, \mathrm{m} / \mathrm{z}(\%): 299\left[\mathrm{M}^{+}, 78 \%\right] ; 222\left[\mathrm{M}^{+}-\mathrm{C}_{6} \mathrm{H}_{5}, 36 \%\right] ; 118\left[\mathrm{C}_{8} \mathrm{H}_{6} \mathrm{O}^{+}\right.$, $100 \%$ ]; $90\left[\mathrm{C}_{7} \mathrm{H}_{6}{ }^{+}, 41 \%\right.$ ]. HRMS m/z [M $\left.{ }^{+}\right]$calcd for $\mathrm{C}_{21} \mathrm{H}_{17} \mathrm{NO}$ : 299.1310; found: 299.1306.

\section{Acknowledgements}

The present research has been financed by Spanish DGCYT (Project CTQ2006-15456-02) and the Generalitat Valenciana for the projects ACOMP07-080. Finally, SCSIE (Universidad de Valencia) is gratefully acknowledged for all the equipment employed

\section{References}

1. (a) Thompson, C. M. Dianion Chemistry in Organic Synthesis. CRC Press: Boca Raton, 1994; pp 88-129. (b) Gil, S.; Parra, M. Curr. Org. Chem. 2002, 6, 283. (c) Gil, S.; Parra, M. Recent Res. Devel. Org. Chem. 2002, 6, 449.

2. Clayden, J. In Organolithiums: selectivity for synthesis; Pergamon Press: Oxford, 2002; p 73.

3. (a) Eames, J.; Suggate, M. J. Angew. Chem. Int. Ed. 2005, 44, 186. (b) Streitwieser, A.; Husemann, M.; Kim, Y-J. J. Org. Chem. 2003, 68, 7937. (c) Sott, R.; Granander, J.; Hilmersson, G. Chem. Eur. J. 2002, 8, 2081. (d) Whisler, M. C.; MacNeil, S.; Snieckus, V.; Beak, P. Angew. Chem. Int. Ed. 2004, 43, 2206.

4. (a) Jarrahpour, A.; Zarei, M. Tetrahedron Lett. 2007, 48, 8712. (b) Ragusa, A.; Rossi, S.; Hayes, J. M.; Stein, M.; Kilburn, J. D. Chem. Eur. J. 2005, 11, 5674.

5. (a) Seebach, D.; Beck, A. K.; Capone, S.; Deniau, G.; Grošelj, U.; Zass, E. Synthesis 2009, 1.

(b) Liu, M.; Sibi, M. P. Tetrahedron 2002, 58, 7991. (c) Davies, S. G.; Smith, A. D.; Price, P. D. Tetrahedron: Asymmetry 2005, 16, 2833.

6. (a) Pousset, C.; Callens, R.; Haddad, M.; Larchevêque, M. Tetrahedron: Asymmetry 2004, 15, 3407. (b) Iijima, K.; Katada, J.; Hayashi, Y. Bioorg. Med. Chem. Lett. 1999, 9, 413.

7. (a) Periasamy, M.; Suresh, S.; Ganesan, S. S. Tetrahedron: Asymmetry 2006, 17, 1323. (b) Luisi, R.; Capriati, V.; Degennaro, L.; Florio, S. Organic Lett. 2003, 5, 2723. (c) Luisi, R.; 
Capriati, V.; Florio, S.; Vista, T. J. Org. Chem. 2003, 68, 9861. (d) Fustero, S.; Pina, B.; Salavert, E.; Navarro, A.; Ramirez de Arellano, M. C.; Simon, A. J. Org. Chem. 2002, 67, 4667.

8. Della Rosa, C.; Gil, S.; Rodriguez, P.; Parra, M. Synthesis 2006, 3092.

9. Hata, S.; Tomioka, K. Tetrahedron 2007, 63, 8514.

10. Silveira, C. C.; Vieira, A. S.; Braga, A. L.; Russowsky, D. Tetrahedron 2005, 61, 9312.

11. Prieto, O.; Lam, H. W. Org. Biomol Chem. 2008, 6, 55.

12. Bellassoued, M.; Arous-Chtara, R.; Mladenova, M.; Kurtev, B.; Gaudemar, M. C.R. Acad. Sc. Paris 1985, 301, 1341.

13. Brun, E. M.; Casades, I.; Gil, S.; Mestres, R.; Parra, M. Tetrahedron Lett. 1998, 39, 5443.

14. Kikuchi, S.; Hashimoto, Y. Heterocycles 2006, 68, 453.

15. Sotoca, E.; Bouillon, J. P.; Gil, S.; Parra, M.; Portella, C. Tetrahedron 2005, 61, 4395.

16. (a) Parra, M.; Mestres, R.; Aparicio, D.; Durana, N.; Rubiales, G. J. Chem. Soc., Perkin Trans I 1989, 327. (b) Brun, E. M.; Gil, S.; Mestres, R.; Parra, M. Synthesis 2000, 273.

17. van Maanen, H. L.; Kleijn, H.; Jastrzebski, J. T. B. H.; Lakin, M. T.; Spek, A. L.; van Koten, G. J. Org. Chem. 1994, 59, 7839.

18. Brittelli, D. R. J. Org. Chem. 1981, 46, 2514. 